\title{
Fusidic acid resistance among clinical isolates of methicillin-resistant Staphylococcus aureus in a Taiwanese hospital
}

\author{
Chih-Ming Chen ${ }^{1 \dagger}$, Mei Huang ${ }^{2 \dagger}$, Huei-Fen Chen ${ }^{3}$, Se-Chin Ke ${ }^{4}$, Chia-Ru Li ${ }^{5}$, Jen-Hsien Wang ${ }^{6,7}$ and Lii-Tzu Wu ${ }^{3 *}$
}

\begin{abstract}
Background: The prevalence of resistance to fusidic acid of methicillin-resistant Staphylococcus aureus (MRSA) was increased each year in a Taiwan hospital. Thirty-four MRSA clinical isolates collected in 2007 and 2008 with reduced susceptibility to FA were selected for further evaluation the presence of resistance determinants.

Results: The most common resistance determinant was fusC, found in 25 of the 34 MRSA isolates. One of the 25 fusidic acid-resistant MRSA harboured both fusB and fusC, which is the first time this has been identified. Mutations in fusA were found in 10 strains, a total of 3 amino-acid substitutions in EF-G (fusA gene) were detected. Two substitutions with $G_{556} S$ and $R_{659} L$ were identified for the first time. Low-level resistance to fusidic acid (MICs, $\leq 32$ $\mathrm{\mu g} / \mathrm{ml}$ ) was found in most our collection. All collected isolates carried type III SCCmec elements. MLST showed the isolates were MRSA ST239. PFGE revealed nine different pulsotypes in one cluster.
\end{abstract}

Conclusions: Our results indicate that the increase in the number of fusidic acid resistant among the MRSA isolates in this hospital is due mainly to the distribution of fusC determinants. Moreover, more than one fusidic acid-resistance mechanism was first detected in a same stain in our collection.

Keywords: fusidic acid MRSA, fusA, fusB, fusC

\section{Background}

The frequently-encountered multi-antibiotic resistance of MRSA has become a major health problem $[1,2]$. The prevalence of MRSA isolates, most of which are health care associated, has slowly increased since 1982, and the appearance and increasing incidence of communityassociated MRSA infections has been documented. Globally, methicillin resistance among nosocomial $S$. aureus isolates is common [3,4].

Fusidic acid has been used to treat infections with $S$. aureus for over 35 years. It is usually used in combination with agents such as vancomycin or rifampin in the treatment of systemic infections caused by MRSA [5]. Fusidic acid inhibits protein synthesis by blocking the elongation of the nascent polypeptide chain through binding to EF$\mathrm{G}$ on the ribosome and preventing the dissociation of EF-

\footnotetext{
* Correspondence: Itwu@mail.cmu.edu.tw

+ Contributed equally

${ }^{3}$ The Institute of Medical Science and Department of Microbiology, China

Medical University and Hospitals, Taichung, Taiwan Full list of author information is available at the end of the article
}

G.GDP from the ribosome [6,7]. The frequency of fusidic acid resistance is not very high; however, the emergence of clinical staphylococcal species that are resistant to fusidic acid has been reported [8-11].

The primary mechanism of fusidic acid resistance in $S$. aureus relates to mutations in fusA, the gene that encodes the ribosomal translocase and translation elongation factor EF-G $[12,13]$. More than 30 different amino acid substitution mutations in fusA have been identified $[12,14,15]$. Subsequently, resistance in natural isolates may also result from the horizontal acquisition of $f u s B$, a poorly characterized plasmid-mediated resistance mechanism [13]. The gene fus $B$ is usually carried by a $21-\mathrm{kb}$ plasmid, pUB101 [16], however, it can also be chromosomal [17]. The fusB gene encodes an inducible protein that protects an in vitro translation system against the inhibitory action of fusidic acid [8]. Recently, two fusB homologues, designated fus $C$ and fus $D$, have been identified in the chromosome of clinical isolates of $S$. aureus and S. saprophyticus, respectively [18]. In addition, fusidic acid-resistant small-colony
C Biomed Central 
variants (SCVs) of S. aureus with mutations in rplF have been designated as FusE mutants [14]. Although frequencies of resistance to fusidic acid have remained generally low, each of these mechanisms has multiple genetic causes, and emerging resistance is a problem that could limit the therapeutic options available for treatment of staphylococcal infections [19].

In this study, a series of MRSA clinical isolates recovered at a regional teaching hospital in middle Taiwan showing fusidic acid MIC $\geq 2 \mu \mathrm{g} / \mathrm{ml}$. The high distribution of fusidic acid resistance determinants fus $C$ was confirmed in MRSA. In addition, different fusidic acid resistance determinants-containing in one isolate was also demonstrated.

\section{Methods}

\section{Bacterial isolates}

From April 2007 to January 2008, 34 clinical isolates of MRSA with fusidic acid resistance were recovered from 34 different patients at Tungs' Taichung MetroHarbor Hospital (TTMHH), a 1405-bed regional teaching hospital in central Taiwan. S. aureus ATCC 29213 and NCTC 8325 have consistently been used as a quality control strain and Pulsed Field Gel Electrophoresis (PFGE) standard strain, respectively. Luria-Bertani (LB) agar and LB broth were used for bacterial growth at $37^{\circ} \mathrm{C}$ with aeration. Mueller-Hinton agar was used for all determinations of minimum inhibitory concentrations (MICs). All isolates were identified on the colony morphology, Gram's stain, a positive catalase reaction and/or results obtained with the phoenix system (BD Diagnostic Systems, Sparks, MD, USA) and frozen at $-80^{\circ} \mathrm{C}$ until used.

\section{Antimicrobial susceptibility tests}

MICs of different antimicrobial agents were determined using the Phoenix Automated Microbiology System (BD Diagnostic Systems, Sparks, MD) and interpreted according to the criteria provided by the Clinical and Laboratory Standards Institute (CLSI). Fusidic acid susceptibility was screened by the disk diffusion method with $10 \mu \mathrm{g}$ fusidic acid containing disks. The interpretive criterion of susceptibility was an inhibition zone $\geq$ $22 \mathrm{~mm}$ in diameter. Fusidic acid MICs were further determined by an agar dilution method following the CLSI guidelines, and susceptibility was categorized using the European Committee for Antimicrobial Susceptibility Testing (EUCAST)/British Society of Antimicrobial Chemotherapy (BSAC) criteria (susceptible, MIC $<2$ $\mu \mathrm{g} / \mathrm{ml}$; resistant, MIC $\geq 2 \mu \mathrm{g} / \mathrm{ml}$ ). The testing MIC range of fusidic acid was $0.12-512 \mu \mathrm{g} / \mathrm{ml}$.

\section{DNA manipulation and PCR}

Total DNA from three to five isolated colonies was prepared using a Wizard genomic DNA preparation kit
(Promega, Madison, WI) with $0.5 \mathrm{mg} / \mathrm{ml}$ of lysostaphin and $0.3 \mathrm{mg} / \mathrm{ml}$ of RNase for the lysis step. The multiplex PCR assay for fus $B$ and fus $C$ used oligonucleotide primers BF (5'-CTATAATGATATTAATGAGATTTTTGG), BR (5'-TTTTTACATATTGACCATCCGAATTGG), CF (5'TTAAAGAAAAAGATATTGATATCTCGG), and CR (5'-TTTACAGAATCCTTTTACTTTATTTGG) to generate amplicons of 431 and 332 bp from the fus $B$ and fus $C$ genes, respectively. The cycling conditions consisted of an initial denaturation step $\left(94^{\circ} \mathrm{C}\right.$ for $\left.3 \mathrm{~min}\right)$, followed by 25 cycles of $94^{\circ} \mathrm{C}(30 \mathrm{~s}), 57^{\circ} \mathrm{C}(30 \mathrm{~s})$ and $72^{\circ} \mathrm{C}(45 \mathrm{~s})$ [20]. For further identification of the fus B and fus C genes, primers FusB-R (5'-ACAGGATCCATTTTCAC AAACATAGT) and FusB-F1(5'-AGGGATCCCATATTTAAAGCTATTG) were used to generate an amplicon comprising the 642 bp fusB with 122 bp of upstream DNA [8], and primers sas0043U (5'-GTAGGATC CATTGGGAATGATAAATAGTGA) and sas0043L (5'TTTGGATCCATCGATTAAGAGTGAGGTACA) were used to generate a $2.5 \mathrm{~kb}$ amplicon with fus $C$ [18]. The fus $A$ gene was PCR-amplified using oligonucleotide primers rpsU and tufL and sequenced with these and three additional primers (AintS1, 5'-TAAGGGTCAGTCATAACTTT; AintS2, 5'-TTCAAAAACAAAGGTGTTCA; and AintS3, 5'-ATGTATTCACGAGGAAC) [20]. The PCR products were electrophoresed in $1.5 \%$ agarose gels and visualized under ultraviolet light. The PCR products were then purified with a commercial kit and both strands of the amplicons were sequenced on an ABI PRISM 370 automated sequencer (PE Applied Biosystems, Franklin Lakes, NJ). Sequence analyses were performed online at the National Center for Biotechnology Information website (http://www.ncbi.nlm.nih.gov).

\section{Southern blot hybridization}

DNA samples were digested by EcoR1 and analyzed by electrophoresis at $30 \mathrm{~V}$ for $2 \mathrm{~h}$ in a $1 \% \mathrm{w} / \mathrm{v}$ agarose gel. The gel was denatured in a solution of $0.5 \mathrm{M}$ $\mathrm{NaOH}$ and $1.5 \mathrm{M} \mathrm{NaCl}$, neutralized in $0.5 \mathrm{M}$ Tris- $\mathrm{HCl}$ $(\mathrm{pH} 7.5)$ and $1.5 \mathrm{M} \mathrm{NaCl}$ on Whatman filter paper (Maidstone, UK), and finally saturated with $10 \% \mathrm{w} / \mathrm{v}$ SDS (15 min for each step). DNA was transferred to a positively charged nylon membrane (Boehringer Mannheim, Mannheim, Germany) using an electrophoretic transfer cell (Bio-Rad Laboratories, Hercules, CA). A probe for fus $C$ was prepared by randomly labelling the $2.5 \mathrm{~kb}$ PCR product of fus $C$ with digoxigenin using a commercial kit (Roche Diagnostics, Mannheim, Germany) according to the manufacturer's instructions. The fus $C$ gene for the hybridization probe was amplified using oligonucleotide primers fusCU 5'GAGG AATATCATATGAATAAAATAGAAGTGTA and fusCL 5'-AGAGTGGATCCCAAAATATAACAAC CCTGATC [18]. 


\section{SCCmec typing by PCR}

The presence of $m e c A$ was determined using the primers MR1 5'-GTGGAATTGGCCAATACAGG and MR2 5'-TGAGTTCTGCAGTACCGGAT, which were used to PCR-amplify a 1,339 bp internal fragment of the gene [21]. PCR was carried out for 30 cycles of $1 \mathrm{~min}$ at $95^{\circ} \mathrm{C}, 1 \mathrm{~min}$ at $55^{\circ} \mathrm{C}$, and $2 \mathrm{~min}$ at $72^{\circ} \mathrm{C}$. Characterization of SCCmec elements was performed by multiple PCR as previously described [22].

\section{PFGE and multilocus sequence typing (MLST)}

Genotyping of $S$. aureus strains was conducted by macrorestriction of bacterial DNA followed by PFGE separation of the resulting fragments. Whole chromosomal DNA of the clinical isolates embedded in agarose gel plugs (FMC Bioproducts, Philadelphia, PA) were treated with proteinase K and SmaI restriction endonuclease according to the manufacturer's recommendations (New England Biolabs, Ipswich, MA). PFGE and DNA fingerprints analysis were performed as described previously [23]. The isolates were also analyzed by MLST as described previously [24].

\section{Plasmid curing}

The clinical isolate with pUB101-like plasmid was subjected to elevated temperature-mediated plasmid elimination by sequential passages in LB (approximately 100 cells into $100 \mathrm{ml}$ ) at $43^{\circ} \mathrm{C}$ with shaking for about 30 generations. Cured strains were diluted and plated on LA plates (LB plus 1\% agar; Merck, Darmstadt, Germany) to obtain single colonies. Loss of cadmium resistance was screened by replica plating at $37^{\circ} \mathrm{C}$ [25]. Loss of the plasmid was confirmed by loss of unselected phenotypic traits (ampicillin resistance) and by PCR of cadXD [15].

\section{Ethics}

This study was reviewed by the Institutional Review Board (IRB) of the TTMHH and it was decided not to constitute the research involving human subject. An exemption certificate was issued by the IRB to attest this fact.

\section{Results}

\section{Isolates and susceptibility tests}

The sources of the 34 fusidic acid-resistant MRSA isolates included sputum $(n=9)$, pus $(n=16)$, blood $(n=$ $5)$, urine $(n=2)$, ascites $(n=1)$, and tip of a central venous catheter $(n=1)$ (Table 1). All 34 clinical isolates were analyzed in more detail with regard to their antibiotic resistance profiles, and they were all susceptible to vancomycin, teicoplanin, quinupristin-dalfopristin, linezolid, and nitrofurantoin. The MICs for fusidic acid $(2-64 \mu \mathrm{g} / \mathrm{ml})$ were low to moderate level resistance phenotype. All isolates were uniformly resistant to penicillin, ampicillin, oxacillin, clindamycin, erythromycin, ciprofloxacin and gentamicin. The susceptible rates and MIC ranges of other antibiotics were as follows: rifampin 91\%; chloramphenicol 88\%; moxifloxacin 6\%; levofloxacin 3\%; tetracycline 3\%; and trimethoprimsulfamethoxazole $3 \%$. The study results revealed that fusidic acid-resistant $S$. aureus was resistant to nearly all tested antibiotics except for vancomycin, teicoplanin, linezolid, nitrofurantoin, quinupristin-dalfopristin, chloramphenicol, and rifampin.

\section{Genetic basis of resistance to fusidic acid: fusB and fusC}

The genetic basis for resistance to fusidic acid in the isolates was determined by a multiplex PCR assay capable of detecting both the $431 \mathrm{bp}$ fusB and $332 \mathrm{bp}$ fus $\mathrm{C}$ genes [20]. Twenty-five of the 34 isolates $(73.5 \%)$ were found to harbour the gene encoding fus $\mathrm{C}$ and one (isolate 32) among the 25 isolates also harboured the gene encoding fus B. Furthermore, using plasmid DNA of isolate 32 as a template, PCR with FusB-specific primers FusB-R1 and FusB-F1 and subsequent sequence analysis of the 764 bp PCR product confirmed the $100 \%$ identity of the fusB gene from plasmid pUB101. A curing study revealed that both the cadXD and fus $\mathrm{B}$ genes were plasmid encoded, and that fus $\mathrm{C}$ remained in the plasmid cured isolate 32. The MIC of fusidic acid for isolate 32 was $8 \mu \mathrm{g} / \mathrm{ml}$ after curing of the plasmid.

The full-length fus $\mathrm{C}$ gene was identified by $\mathrm{PCR}$ and sequenced in isolates $4,24,29,30$, and 32 . The alignment of the amino acid sequences deduced from these isolates 4, 24, 30, and 32 fusC DNA sequences revealed $100 \%$ identity with FusC protein of S. aureus MSSA476 [18]. However, fus $C$ from isolate 29 carried a nonsense mutation (S175 was encoded by TAA rather than TCA) that produced a change from fusidic acid resistance $(\mathrm{MIC}=8 \mu \mathrm{g} / \mathrm{ml})$ to fusidic acid susceptibility $(\mathrm{MIC}<$ $0.125 \mu \mathrm{g} / \mathrm{ml}$ ) following two non-selective subcultures. The other isolates were screened for the presence of the fusC gene by Southern hybridization, and all tested isolates were positive for fus C (Figure 1).

\section{Detection of fusA gene mutations}

PCR amplification and complete sequencing were performed to detect fusA gene mutations in the 34 isolates (Table 1). Five isolates possessed a mutation in $\mathrm{H}_{457} \mathrm{Y}$, two isolates (isolates 9 and 33) exhibited a $\mathrm{G}_{556} \mathrm{~S}$ mutation, and two isolates (isolates 10 and 21) harboured mutations in $\mathrm{H}_{457} \mathrm{Y}$ and $\mathrm{G}_{556} \mathrm{~S}$. In addition, isolate 31 possessed a mutation in $\mathrm{H}_{457} \mathrm{Y}$ and $\mathrm{R}_{659} \mathrm{~L}$. Single amino acid substitutions were found in seven isolates, and two amino acid substitutions were found in the other three. This is the first time that two different amino acid substitutions, $\mathrm{G}_{556} \mathrm{~S}$ and $\mathrm{R}_{659} \mathrm{~L}$, have been reported in fus $\mathrm{A}$ 
Table 1 Characteristics and mechanisms of the $\mathbf{3 4}$ fusidic acid-resistant MRSA clinical isolates

\begin{tabular}{|c|c|c|c|c|c|c|c|c|c|}
\hline Isolate & Specimen & $\begin{array}{l}\text { FA MIC } \\
(\mu \mathrm{g} / \mathrm{ml})\end{array}$ & $\begin{array}{c}\text { VAN MIC } \\
(\mu \mathrm{g} / \mathrm{ml})\end{array}$ & $\begin{array}{c}\text { LZD MIC } \\
(\mu \mathrm{g} / \mathrm{ml})\end{array}$ & $\begin{array}{c}\text { OXA MIC } \\
(\mu \mathrm{g} / \mathrm{ml})\end{array}$ & $\begin{array}{l}\text { RIF MIC } \\
(\mu \mathrm{g} / \mathrm{ml})\end{array}$ & fusC & Polymorphism(s) in EF-G & PFGE patterns \\
\hline 1 & Pus & 8 & 1 & 1 & $>2$ & $<=0.5$ & - & $\mathrm{H}_{457} \mathrm{Y}$ & $\mathrm{A} 1$ \\
\hline 2 & Pus & 32 & 1 & 2 & $>2$ & $<=0.5$ & + & - & $\mathrm{A} 1$ \\
\hline 3 & Pus & 8 & 1 & 1 & $>2$ & $<=0.5$ & + & - & $\mathrm{A} 1$ \\
\hline 4 & Sputum & 16 & 1 & 2 & $>2$ & $<=0.5$ & + & $\mathrm{H}_{457} \mathrm{Y}$ & $\mathrm{A} 1$ \\
\hline 5 & Sputum & 32 & 2 & 2 & $>2$ & $>2$ & + & - & $\mathrm{A} 1$ \\
\hline 6 & Pus & 16 & 1 & 1 & $>2$ & $>2$ & + & - & $\mathrm{A} 2$ \\
\hline 7 & Pus & 8 & 1 & 1 & $>2$ & $<=0.5$ & + & - & A3 \\
\hline 8 & Sputum & 16 & 1 & 1 & $>2$ & $<=0.5$ & + & - & $\mathrm{A} 3$ \\
\hline 9 & Pus & 16 & 1 & 1 & $>2$ & $<=0.5$ & - & $\mathrm{G}_{556} \mathrm{~S}$ & A3 \\
\hline 10 & Sputum & 16 & 1 & 1 & $>2$ & $<=0.5$ & - & $\mathrm{H}_{457} \mathrm{Y}, \mathrm{G}_{556} \mathrm{~S}$ & $\mathrm{~A} 3$ \\
\hline 11 & Ascites & 8 & 1 & 1 & $>2$ & $<=0.5$ & - & $\mathrm{H}_{457} \mathrm{Y}$ & $\mathrm{A} 3$ \\
\hline 12 & Pus & 64 & 2 & 2 & $>2$ & $<=0.5$ & + & - & $\mathrm{A} 3$ \\
\hline 13 & Sputum & 64 & 2 & 2 & $>2$ & $<=0.5$ & - & $\mathrm{H}_{457} \mathrm{Y}$ & A3 \\
\hline 14 & Pus & 16 & 1 & 1 & $>2$ & $<=0.5$ & + & - & A3 \\
\hline 15 & Blood & 4 & 1 & 1 & $>2$ & $<=0.5$ & + & - & A3 \\
\hline 16 & Pus & 8 & 1 & 1 & $>2$ & $<=0.5$ & + & - & A3 \\
\hline 17 & Blood & 8 & 1 & 1 & $>2$ & $<=0.5$ & + & - & $\mathrm{A} 3$ \\
\hline 18 & Blood & 16 & 1 & 1 & $>2$ & $<=0.5$ & + & - & A3 \\
\hline 19 & Blood & 16 & 1 & 1 & $>2$ & $<=0.5$ & + & - & A3 \\
\hline 20 & Pus & 2 & 2 & 1 & $>2$ & $<=0.5$ & + & - & A3 \\
\hline 21 & Urine & 2 & 2 & 2 & $>2$ & $<=0.5$ & - & H457Y, G556S & A3 \\
\hline 22 & Sputum & 2 & 2 & 2 & $>2$ & $<=0.5$ & + & - & A3 \\
\hline 23 & Pus & 16 & 2 & 1 & $>2$ & $>2$ & - & H457Y & A4 \\
\hline 24 & Pus & 2 & 1 & 1 & $>2$ & $<=0.5$ & + & - & A5 \\
\hline 25 & Urine & 16 & 1 & 1 & $>2$ & $<=0.5$ & + & - & A6 \\
\hline 26 & CVP tip & 8 & 1 & 2 & $>2$ & $<=0.5$ & + & - & A6 \\
\hline 27 & Pus & 2 & 2 & 2 & $>2$ & $<=0.5$ & + & - & A6 \\
\hline 28 & Sputum & 16 & 1 & 2 & $>2$ & $<=0.5$ & + & - & A7 \\
\hline $29^{a}$ & Pus & 8 & 1 & 2 & $>2$ & $<=0.5$ & + & - & A8 \\
\hline 30 & Sputum & 16 & 1 & 2 & $>2$ & $<=0.5$ & + & - & A9 \\
\hline 31 & Pus & 16 & 1 & 2 & $>2$ & $<=0.5$ & - & H457Y, R659L & A9 \\
\hline 32 & Sputum & 8 & 1 & 2 & $>2$ & $<=0.5$ & + & - & A9 \\
\hline 33 & Blood & 16 & 1 & 1 & $>2$ & $<=0.5$ & - & G556S & A9 \\
\hline 34 & Pus & 2 & 2 & 2 & $>2$ & $<=0.5$ & + & - & A9 \\
\hline
\end{tabular}

FA, fusidic acid; VAN, vancomycin; LZD, linezolid; OXA, oxacillin; RIF, rifampin

gene mutations. Furthermore, one isolate (isolate 4) was encoded with fus $C$ and fusA gene mutation. In this study, the most common amino acid substitution $\mathrm{H}_{457} \mathrm{Y}$ did not result in a high level of fusidic acid resistance $(\mathrm{MIC} \geq 128 \mu \mathrm{g} / \mathrm{ml})$.

\section{Molecular epidemiological analysis}

All 34 isolates included in this study met the criteria of being health care associated. The genotype analyses and their frequencies are shown in Table 1. Only one defined MLST type (ST239) was evident. All 34 isolates carried SCCmec type III elements. PFGE patterns of SmaI macrorestriction fragment analysis of these 34 isolates revealed nine distinct pulsotypes (A1-A9) that were classified into one cluster ( $>80 \%$ similarity) (Figure 2 ). The results of PFGE patterns are summarized in Table 1.

\section{Discussion}

Previous studies of fusidic acid-resistance in clinical isolates have mostly focused on methicillin-susceptible $S$. aureus (MSSA) and other staphylococci $[17,20,26]$. Chen et al. recently reported that the prevalence of fusidic acid-resistance determinants was quite different between MRSA and MSSA groups [27]. In northern Taiwan collections, the fusA mutations were the major determinant (84\%) followed by fusC with $16 \%$ fusidic acid-resistance in MRSA isolates [27]. In the present study based in central Taiwan, we found that the fusidic acid-resistant 


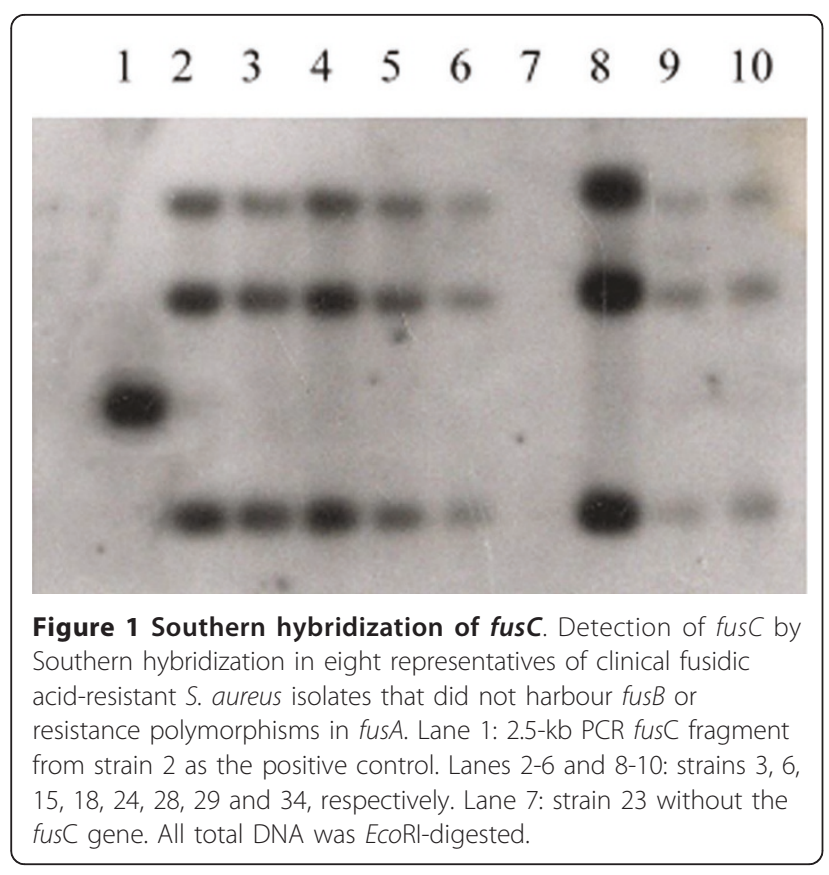

predominant determinant in MRSA was a high prevalence of fus $\mathrm{C}$ with $74 \%$ in clinical isolates. Furthermore, one isolate carried the fus B determinant on the plasmid and fus $\mathrm{C}$ determinant on the chromosome in a clinical fusidic acid-resistant $S$. aureus isolate. The FusC protein has a $45 \%$ amino acid similarity to FusB. The fusC gene was originally identified in the genome sequence of $S$. aureus MSSA476, and has been reported in fusidic acidresistant S. intermedius and S. epidermidis $[18,20]$. In most European collections, fus $C$ has been shown to be responsible for resistance to fusidic acid in all $S$. aureus strains examined that do not carry $f u s B$ or resistance mutations in fusA $[17,18]$. Moreover, the fus $B$ gene has only been detected in MSSA, not in MRSA in most clinical collections in Taiwan [27]. Therefore, the present study shows the spread of fusC in Taiwan and for the first time demonstrates the presence of both $f u s B$ and fus $C$ in a MRSA clinical isolate.

The most common mutation in fusA that conferred resistance to fusidic acid was the substitution $\mathrm{H}_{457} \mathrm{Y}$ in our study (Table 1). We reviewed the English literature and did not find any reports of two amino acid substitutions in EF-G of $G_{556} S$ and $R_{659} L$ relative to the resistance of fusidic acid. Mutations in EF-G are associated with fitness cost in the fusidic acid-resistance of $S$. aureus in vitro and in vivo [12,14]. The resistance mutations with amino acid substitutions occur mostly in structural domain III of EF-G, but some occur in domains I and V $[28,29]$. We identified a novel substitution present in fusidic acid-resistant $S$. aureus (isolates 9 and 33), which conferred an identical resistance mutation in fusA $\left(\mathrm{G}_{556} \mathrm{~S}\right)$. The two isolates exhibited resistance to fusidic acid with $\mathrm{MIC}=16 \mu \mathrm{g} / \mathrm{ml}$ and carried neither fusB nor fusC. In addition, substitution $\mathrm{G}_{556} \mathrm{~S}$ was found in isolates 10 and 21 and was accompanied by mutations in fusA $\left(\mathrm{H}_{457} \mathrm{Y}\right)$. Another novel substitution amino acid substitution $\mathrm{R}_{659} \mathrm{~L}$ located in domain V of EF-G was found to be accompanied with fus $C$ mutations in our study. The role of this newly found amino acid substitution in fusA on the level of resistance is unknown and needs further investigation. Of the 34 isolates that were studied completely, isolate 4 harboured fus C and a resistance mutation in fusA $\left(\mathrm{H}_{457} \mathrm{Y}\right)$. This indicates that the fusidic acid-resistance in these MRSA clinical isolates had multiple genetic lineages.

The isolates with fusB and fusC determinants usually displayed higher level resistance to fusidic acid (> $16 \mu \mathrm{g}$ / $\mathrm{ml})[8,17]$. The MICs of fusidic acid in our collections carrying fus $C$ ranged from $2-64 \mu \mathrm{g} / \mathrm{ml}$. It is not clear the reason why in non-selective subcultures, isolate 29 with one mutation site of the fus $C$ gene lost the resistance to fusidic acid. We hypothesized that the mutation may result in FusC truncated after amino acid 174, and thus isolate 29 became susceptible. In this study, the single-amino-acid substitutions in EF-G substitution did not result in a high level fusidic acid resistance which is similar to previous report in MRSA strains belonging to $\mathrm{CC} 8, \mathrm{H}_{457} \mathrm{Y}$ mutation was associated with MIC of 64 $\mu \mathrm{g} / \mathrm{L}$ and $\mathrm{H}_{457} \mathrm{Q}$ was associated with MIC of $4 \mu \mathrm{g} / \mathrm{L}$ [30]. The level of fusidic acid resistance in the isolate 4 with two fusidic acid resistance determinants couldn't be accounted for by their genotypes when compared with other clinical isolates with one of the determinants. A previous study showed a similar result that a laboratory strain containing both fus $A$ resistance mutation and fus $B$ failed to increase the level of fusidic acid resistance [17]. The chromosomal gene fus $C$ confer resistance to fusidic acid on S. aureus or S. intermedius is identified with $45 \%$ amino acid similarity to FusB, protect EF-G from the antibiotic [18]. Genes for FusB-type resistance (fusB and fusC) are thought to act by the same mechanism of protection the drug target [18]. It remains unclear whether these resistance mechanisms of a strain do act in combination or not. The precise action mode of FusB-type resistance awaits further investigation. The level of fusidic acid resistance in isolate 32 did not decrease after curing the pUB101 plasmid. The result may indicate that the resistance mechanisms do not act synergistically or additively.

In this study, all MRSA isolates met the criteria of being health-care associated. PFGE patterns revealed that there was greater than $80 \%$ similarity among the isolates. MLST and SCCmec typing showed that all isolates belonged to ST239 and carried SCCmec III elements, which is the most prevalent health care- 


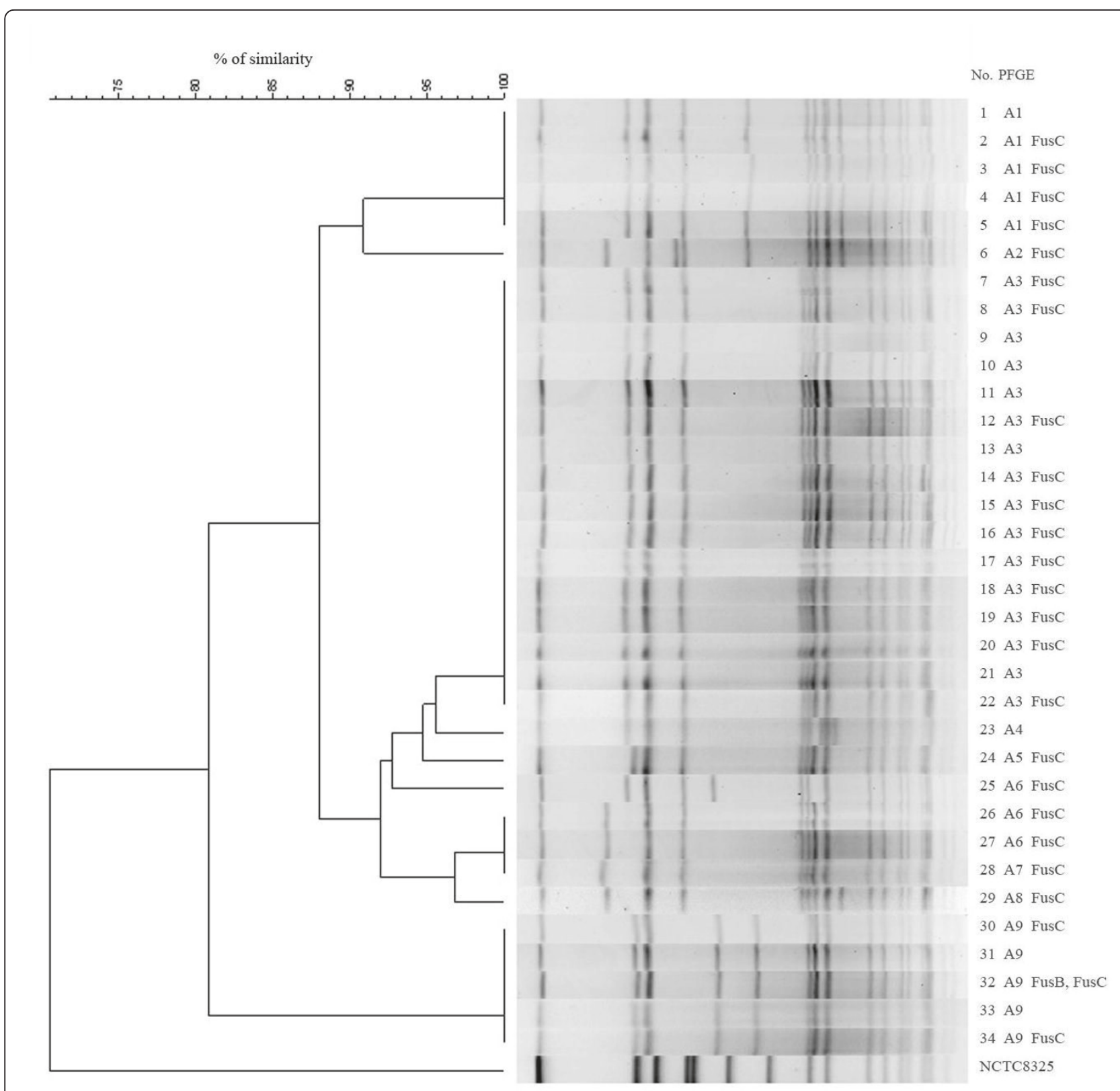

Figure 2 Smal PFGE patterns of the 34 clinical fusidic acid-resistant Staphylococcus aureus isolates. PFGE patterns analysis of these 34 isolates revealed nine distinct pulsotypes (A1-A9) that were classified into one cluster.

associated strain of MRSA in Taiwan [31]. A previous study conducted in 2002-2007 in northern Taiwan also revealed that most of fusidic acid-resistant MRSA isolates carried SCCmec type III [27]. The two studies results suggest that a clonal strain had disseminated in Taiwan during the period of the study. In contrast to our findings, a previous European study finding indicated that the majority of fusidic acid-resistant MRSA isolates belonged to CC80-MRSA-IV clone carrying fusB and $\mathrm{CC} 5$ clone harbouring fusC [30].

\section{Conclusion}

In conclusion, we hypothesize that the prevalence of fusidic acid-resistance in S. aureus was commonly associated with the fusC determinant in our isolates. It is interesting to note that some studied isolates possessed more than one fusidic acid-resistance mechanism in our collection. The fus $C$ and acquired FusB-family determinants in a single isolate were first detected and one isolate with fusC also carried a fus A mutation in $\mathrm{H}_{457} \mathrm{Y}$. Phylogenetic analysis clearly demonstrated the spread of 
a major clonal strain of fusidic acid-resistant MRSA in our institution. Due to the concern of clonal spread and growing expansion of fusidic acid-resistant determinants, particularly FusC in MRSA, large-scale, prospective surveillance monitoring for fusidic acid-resistance in $S$. aureus and MRSA is now ongoing in Taiwan.

\section{Abbreviations}

BSAC: British Society of Antimicrobial Chemotherapy; CLSI: Clinical and Laboratory Standards Institute; EUCAST: European Committee for Antimicrobial Susceptibility Testing; FA: fusidic acid; IRB: Institutional Review Board; LB: Luria-Bertani; MICs: minimum inhibitory concentrations; MLST: multilocus sequence typing; MRSA: methicillin-resistant Staphylococcus aureus; MSSA: methicillin-susceptible S. aureus; PCR: polymerase chain reaction; PFGE: pulsed field gel electrophoresis; SCC: staphylococcal chromosome cassette; SCVs: small-colony variants; TTMHH: Tungs' Taichung MetroHarbor Hospital.

\section{Acknowledgements}

We wish to thank Chien-Shun Chiou of the third branch office of Centers for Diseases Control of Taiwan for his assistance in PFGE analysis. This work was supported in part by research grant CMU97-104 from the China Medical University.

\section{Author details}

'Division of Infectious Disease, Department of Internal Medicine, Tungs' Taichung MetroHarbor Hospital, Taiwan. ${ }^{2}$ Division of Infectious Disease, Show Chwan Memorial Hospital, Taiwan. ${ }^{3}$ The Institute of Medical Science and Department of Microbiology, China Medical University and Hospitals, Taichung, Taiwan. ${ }^{4}$ Infection Control Office, Tungs' Taichung MetroHarbor Hospital, Taiwan. ${ }^{5}$ Department of Medical Research, Tungs' Taichung MetroHarbor Hospital, Taiwan. ${ }^{6}$ Department of Internal Medicine and Section of Clinical Microbiology, China Medical College-Hospital, Taichung, Taiwan. ${ }^{7}$ Department of Laboratory Medicine, China Medical College-Hospital, Taichung, Taiwan.

\section{Authors' contributions}

CMC planned the idea and prepared the manuscript. MH participated in the study design and provided resources of experimental work. HFC conducted the experimental work. SCK and CRL provided technical help with PFGE and MLST. JHW supervised study design. LTW conceived this study, participated in its design, and the coordination and writing of the manuscript. All authors read and approved the final manuscript.

\section{Competing interests}

The authors declare that they have no competing interests.

Received: 19 December 2010 Accepted: 12 May 2011 Published: 12 May 2011

\section{References}

1. Kluytmans J, van Belkum A, Verbrugh H: Nasal carriage of Staphylococcus aureus: epidemiology, underlying mechanisms, and associated risks. Clin Microbiol Rev 1997, 10(3):505-520.

2. Livermore DM: Antibiotic resistance in staphylococci. Int I Antimicrob Agents 2000, 16(Suppl 1):S3-10

3. Grundmann H, Aires-de-Sousa M, Boyce J, Tiemersma E: Emergence and resurgence of meticillin-resistant Staphylococcus aureus as a publichealth threat. Lancet 2006, 368(9538):874-885.

4. Gould SW, Rollason J, Hilton AC, Cuschieri P, MCAuliffe L, Easmon SL, Fielder MD: UK epidemic strains of meticillin-resistant Staphylococcus aureus in clinical samples from Malta. J Med Microbiol 2008, 57(Pt 11):1394-1398.

5. Whitby M: Fusidic acid in the treatment of methicillin-resistant Staphylococcus aureus. Int J Antimicrob Agents 1999, 12(Suppl 2):S67-71.

6. Bodley JW, Zieve FJ, Lin L, Zieve ST: Formation of the ribosome-G factorGDP complex in the presence of fusidic acid. Biochem Biophys Res Commun 1969, 37(3):437-443.
7. Gao YG, Selmer M, Dunham CM, Weixlbaumer A, Kelley AC, Ramakrishnan $\mathrm{V}$ : The structure of the ribosome with elongation factor $\mathrm{G}$ trapped in the posttranslocational state. Science 2009, 326(5953):694-699.

8. O'Neill AJ, Chopra I: Molecular basis of fusB-mediated resistance to fusidic acid in Staphylococcus aureus. Mol Microbiol 2006, 59(2):664-676.

9. O'Neill AJ, Larsen AR, Skov R, Henriksen AS, Chopra I: Characterization of the epidemic European fusidic acid-resistant impetigo clone of Staphylococcus aureus. J Clin Microbiol 2007, 45(5):1505-1510.

10. Woodford N, Afzal-Shah M, Warner M, Livermore DM: In vitro activity of retapamulin against Staphylococcus aureus isolates resistant to fusidic acid and mupirocin. J Antimicrob Chemother 2008, 62(4):766-768.

11. Osterlund A, Kahlmeter G, Haeggman S, Olsson-Liljequist B: Staphylococcus aureus resistant to fusidic acid among Swedish children: a follow-up study. Scand J Infect Dis 2006, 38(5):334-334.

12. Nagaev I, Bjorkman J, Andersson DI, Hughes D: Biological cost and compensatory evolution in fusidic acid-resistant Staphylococcus aureus. Mol Microbiol 2001, 40(2):433-439.

13. Turnidge J, Collignon P: Resistance to fusidic acid. Int J Antimicrob Agents 1999, 12(Suppl 2):S35-44.

14. Norstrom T, Lannergard J, Hughes D: Genetic and phenotypic identification of fusidic acid-resistant mutants with the small-colonyvariant phenotype in Staphylococcus aureus. Antimicrob Agents Chemother 2007, 51(12):4438-4446.

15. Lannergard J, Norstrom T, Hughes D: Genetic determinants of resistance to fusidic acid among clinical bacteremia isolates of Staphylococcus aureus. Antimicrob Agents Chemother 2009, 53(5):2059-2065.

16. O'Brien FG, Price C, Grubb WB, Gustafson JE: Genetic characterization of the fusidic acid and cadmium resistance determinants of Staphylococcus aureus plasmid pUB101. J Antimicrob Chemother 2002, 50(3):313-321.

17. O'Neill AJ, Larsen AR, Henriksen AS, Chopra I: A fusidic acid-resistant epidemic strain of Staphylococcus aureus carries the fusB determinant, whereas fusA mutations are prevalent in other resistant isolates. Antimicrob Agents Chemother 2004, 48(9):3594-3597.

18. O'Neill AJ, McLaws F, Kahlmeter G, Henriksen AS, Chopra I: Genetic basis of resistance to fusidic acid in staphylococci. Antimicrob Agents Chemother 2007, 51(5):1737-1740

19. Dobie D, Gray J: Fusidic acid resistance in Staphylococcus aureus. Arch Dis Child 2004, 89(1):74-77.

20. McLaws F, Chopra I, O'Neill AJ: High prevalence of resistance to fusidic acid in clinical isolates of Staphylococcus epidermidis. J Antimicrob Chemother 2008, 61(5):1040-1043.

21. Weller TM: The distribution of $m e c A, \operatorname{mec} R 1$ and $m e c l$ and sequence analysis of $\mathrm{mecl}$ and the mec promoter region in staphylococci expressing resistance to methicillin. J Antimicrob Chemother 1999, 43(1):15-22.

22. Oliveira DC, de Lencastre H: Multiplex PCR strategy for rapid identification of structural types and variants of the mec element in methicillinresistant Staphylococcus aureus. Antimicrob Agents Chemother 2002, 46(7):2155-2161

23. McDougal LK, Steward CD, Killgore GE, Chaitram JM, McAllister SK Tenover FC: Pulsed-field gel electrophoresis typing of oxacillin-resistant Staphylococcus aureus isolates from the United States: establishing a national database. J Clin Microbiol 2003, 41(11):5113-5120.

24. Enright MC, Day NP, Davies CE, Peacock SJ, Spratt BG: Multilocus sequence typing for characterization of methicillin-resistant and methicillinsusceptible clones of Staphylococcus aureus. J Clin Microbiol 2000, 38(3):1008-1015

25. Lacey RW, Grinsted J: Linkage of fusidic acid resistance to the penicillinase plasmid in Staphylococcus aureus. J Gen Microbiol 1972, 73(3):501-508

26. Osterlund A, Eden T, Olsson-Liljequist B, Haeggman S, Kahlmeter G: Clonal spread among Swedish children of a Staphylococcus aureus strain resistant to fusidic acid. Scand J Infect Dis 2002, 34(10):729-734.

27. Chen HJ, Hung WC, Tseng SP, Tsai JC, Hsueh PR, Teng LJ: Fusidic Acid Resistance Determinants in Staphylococcus aureus Clinical Isolates. Antimicrob Agents Chemother 2010, 54(12):4985-4991.

28. Laurberg M, Kristensen O, Martemyanov K, Gudkov AT, Nagaev I, Hughes D, Liljas A: Structure of a mutant EF-G reveals domain III and possibly the fusidic acid binding site. J Mol Biol 2000, 303(4):593-603.

29. Chen Y, Koripella RK, Sanyal S, Selmer M: Staphylococcus aureus elongation factor G-structure and analysis of a target for fusidic acid. FEBS J 2010, 277(18):3789-3803. 
30. McLaws FB, Larsen AR, Skov RL, Chopra I, O'Neill AJ: Distribution of Fusidic Acid Resistance Determinants in Methicillin-Resistant Staphylococcus aureus. Antimicrob Agents Chemother 2011, 55(3):1173-1176.

31. Huang YH, Tseng SP, Hu JM, Tsai JC, Hsueh PR, Teng LJ: Clonal spread of SCCmec type IV methicillin-resistant Staphylococcus aureus between community and hospital. Clin Microbiol Infect 2007, 13(7):717-724.

doi:10.1186/1471-2180-11-98

Cite this article as: Chen et al:: Fusidic acid resistance among clinical isolates of methicillin-resistant Staphylococcus aureus in a Taiwanese hospital. BMC Microbiology 2011 11:98.

Submit your next manuscript to BioMed Central and take full advantage of:

- Convenient online submission

- Thorough peer review

- No space constraints or color figure charges

- Immediate publication on acceptance

- Inclusion in PubMed, CAS, Scopus and Google Scholar

- Research which is freely available for redistribution

Submit your manuscript at www.biomedcentral.com/submit
() Biomed Central 